\title{
Double Puncture-Aspiration-Injection-Reaspiration (dPAIR) in Hydatid Cysts Management: Report of 10 Cases
}

\author{
S. Bellasri ${ }^{1 *}$, B. Slioui ${ }^{1}$, R. Akka ${ }^{2}$, H. Baba ${ }^{3}$, M. Sbai ${ }^{4}$, N. Hammoune ${ }^{1}$, A. Mouhsine ${ }^{1}$, E. Atmane $^{1}$
}

\author{
${ }^{1}$ Interventional Radiology Department, Avicenne Military Hospital, CadiAyyad University, Morocco \\ ${ }^{2}$ Hepatology Department, Avicenne Military Hospital, CadiAyyad University, Morocco \\ ${ }^{3}$ Surgery Department, Avicenne Military Hospital, CadiAyyad University, Morocco \\ ${ }^{4}$ Parasitology Department, Avicenne Military Hospital, CadiAyyad University, Morocco
}

Introduction: Hydatid cyst is endemic parasitic zoonosis in North Africa, caused by Echinococcus granulosus. The cysts commonly developed in liver. Classically the treatment is based on association of open surgery and oral chemotherapy, but since the mid 1980s a minimally invasive procedure was proposed by a Tunisian team, called PAIR (Puncture, Aspiration, Injection, Re-aspiration). This technique proved its efficacy and safety management of active hydatid cysts. Aims and scope: We report a reinforced variant of this procedure with double aspiration, injection and re-aspiration using initially hypertonic saline serum and secondary absolute alcohol $96 \%$. Material and methods: A retrospective study conducted on 8 patients (10 liver hydatid cysts) collected from the department of interventional radiology between jan-2016 to decem-2020. An 18 needle puncture after local anaesthesia and under sonographic guidance, aspiration of the cyst and subsequent injection aspiration of two protoscolicidal agent: hypertonic saline serum first and then absolute alcohol 96\%. Results: All cases were successfully treated, with short hospitalization length. There was no major complication or morbidity. Conclusions: Obviously, this second aspiration-injection, reaspiration increase efficacy and decrease recurrence probability.

Keywords: Hydatid cyst, Puncture, Aspiration, Injection, Re-aspiration, Double PAIR.

Copyright $(\mathcal{C} 2021$ The Author(s): This is an open-access article distributed under the terms of the Creative Commons Attribution 4.0 International License (CC BY-NC 4.0) which permits unrestricted use, distribution, and reproduction in any medium for non-commercial use provided the original author and source are credited.

\section{INTRODUCTION}

Hydatid disease is a common parasitic infection due to larval stage of Echinococcus granulosus and is endemic in many parts of the world such as Mediterranean, Africa...Where animal husbandry is frequent. It affects the liver more than other organs and can cause serious intra and extra hepatic complications with increased morbidity and mortality $[1,2]$.

Open surgery associated with chemotherapy was the treatment of choice, but in 1986, a mini invasive procedure was proposed by a Tunisian team, called PAIR (puncture, aspiration, injection, reaspiration). The effectiveness and safety of PAIR was demonstrated in medical literature and from a worldwide collection of data and has been indicated by the world health organisation "WHO" as a treatment of hydatid cyst type I, II and III of Gharbi classification [3-7].

We presented our experience using a variant of PAIR that consists of a double aspiration injection re- aspiration using two protoscolicides (hypertonic saline serum $20 \%$ and secondary absolute alcohol 96\%). We believe that this variant can increase the efficacy of the procedure and reduce the risk of recurrence. We highlight the safety and effectiveness of this minimally invasive management of hydatid cyst.

\section{Materials And Methods}

The present study was conducted on 8 patients collected from the department of interventional radiology from jan-2016 to decem-2020. A total of 10 non complicated cysts were collected irrespective of age, sex (two patients were treated subsequently for double cysts).

Inclusion criteria were: single or multiple hydatid cysts type I or II of Gharbi classification, adequate liver parenchyma windows $(\geq 1 \mathrm{~cm})$, and patients who refused surgery and relapsed after surgery.

Type III, IV and V of the classification of Gharbi, Non-cooperative patients and inaccessible or risky location of the cyst in the liver were excluded. 
Prophylactic chemotherapy with albendazole $(10 \mathrm{mg} / \mathrm{kg}) 1$ week before the procedure was started.

All procedures were performed by an experimented interventional radiologist using a percutaneous technique guided by sonography or CT imaging supported by a person trained in reanimation with basic resuscitation equipment and intravenous line.

All patients were informed consent about risks. Light sedation and local anaesthesia were applied.

We used $18 \mathrm{G}$ needle for puncture under sonographic guidance (GE, P9, USA), we examined the color and content of the aspired fluid to rule out biliary fistula. Aspiration of $2 / 3$ of the cyst (to be sure that the extremity of the needle won't go outside the cyst) and injection of ( $1 / 3$ of the amount of aspirated volume) of saline serum hypertonic $20 \%$. We re-aspirated the saline serum after $10 \mathrm{~min}$, and then we injected the same volume of $95 \%$ alcohol. Finally we re-aspirated the alcohol $10 \mathrm{~min}$ later.

A 7F pig's tail catheter (Boston Scientific, Quincy, MA, USA) was used in large cyst (more than $5 \mathrm{~cm}$ ) with aspiration of total volume of the cyst, and CT cystography was realised in 5 patients.

Size of cyst, location in liver, age, number, time of procedure, and follow up were registred.
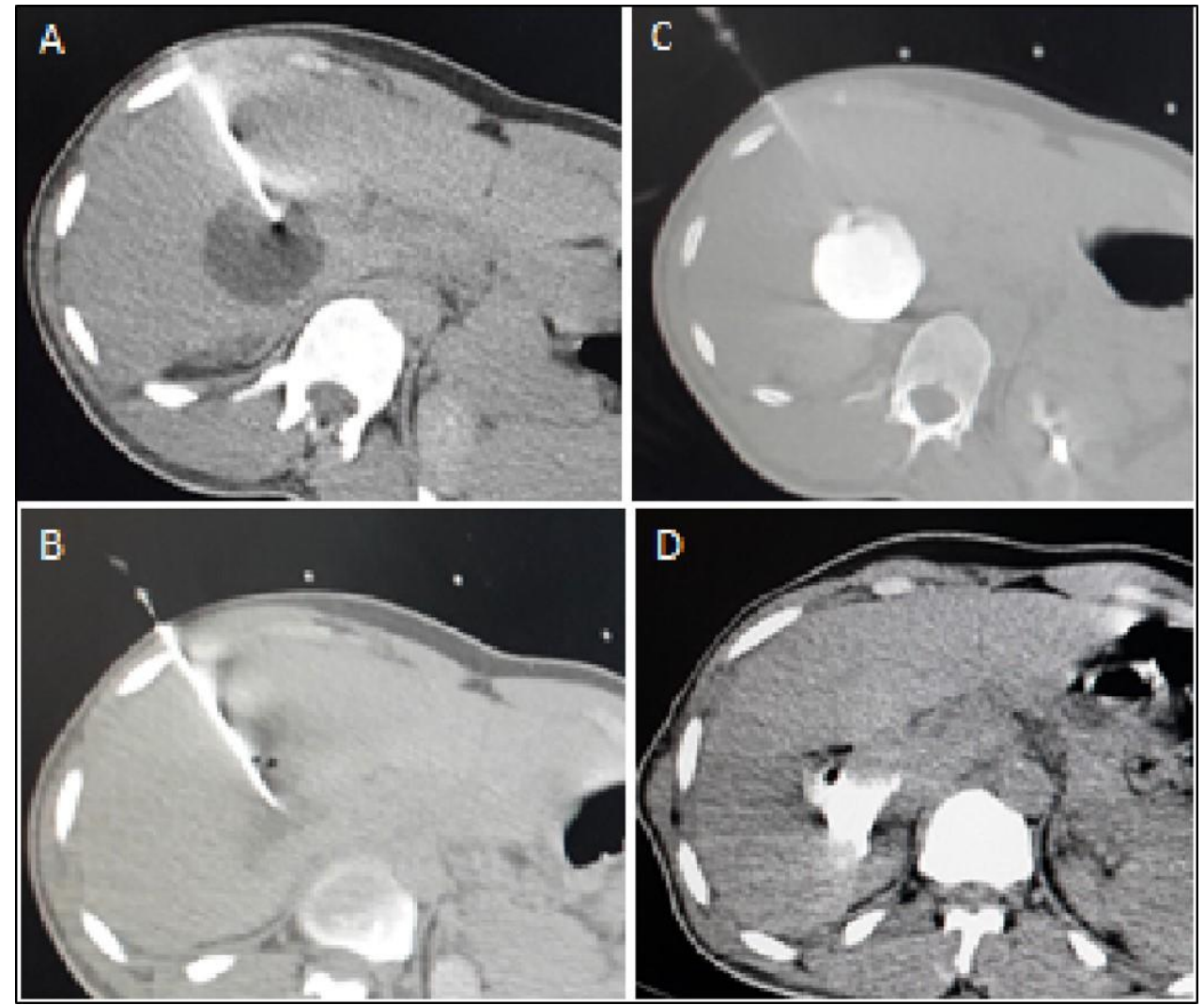

Fig-1: A 24years old man with deep location of hydatid liver cyst measuring $42 \mathrm{~cm}$ in size, treated by double PAIR procedure using computed tomographyCT guidance: (A) Puncture with 18G needle. (B) Aspiration. (C) Injection of hypertonic saline serum $20 \%$ followed by 6cc contrast media (cystography). (D) Reaspiration 

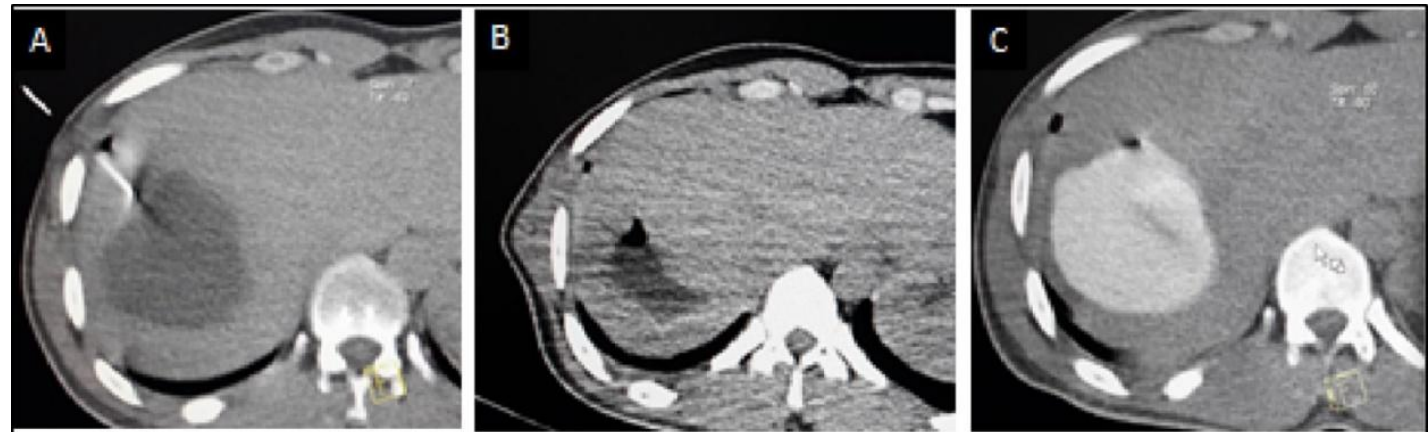

Fig-2: Axial CT slices of the liver of 41 years old woman with Gharbi type I hydatid cyst located in segment VII, treated by double PAIR: (A) Puncture. (B) Aspiration. (C) Injection of hypertonic saline serum with cystography to be sure that there is no biliary fistula

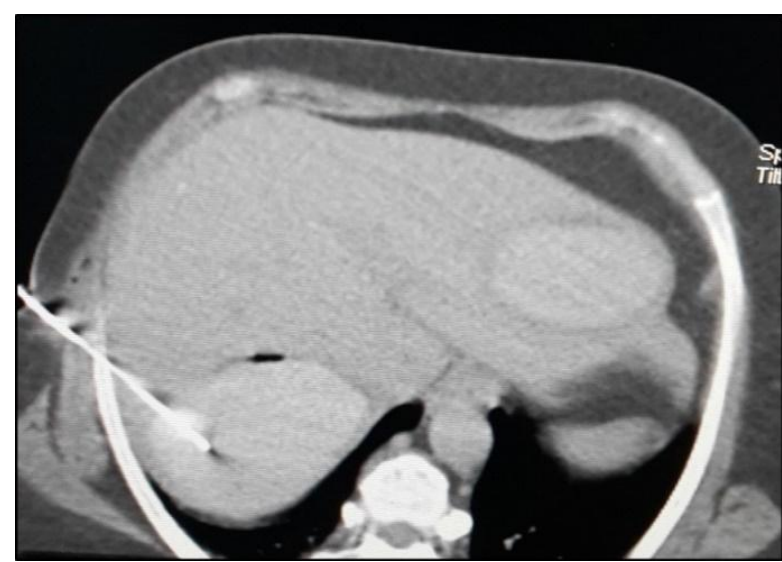

Fig-3: CT scan emphasised simultaneous Gharbi type I hydatid cyst treated subsequently by double PAIR with cystography. Cysts located in segments VII and II of the liver

\section{DiSCUSSION}

Hydatid disease is a major health problem in endemic areas, and has spread with immigration to many other parts of the world [7]. Ultrasound is the primary imaging modality choice which allow diagnosis, classification, and guidance of minimally therapeutic procedure [6]. The traditional treatment of hydatid disease is surgery and /or oral chemotherapy. The overall mortality rate after surgery ranges from $0 \%$ to $6.3 \%$ with various surgical procedures. The objectives of the surgical treatment of hydatid cysts are to inactivate the parasites, evacuate the cyst cavity, remove the germinal layer, and obliterate the residual cavity. Postsurgical complications vary between $12.5 \%$ and $80 \%$ depending on characteristics of cyst and surgical procedures. Percutaneous treatment serve the same purpose and is very effective and has good results when compared with surgery for Gharbi type I-III cysts [8]. Thousands of cases with hydatid cyst have been treated by PAIR which consolidates the safety and efficacy of this technique in comparison with surgery.

Medical treatment should be used for prophylaxis against spillage at any intervention and reduce risk of recurrence $[9,10]$. In our cases, we started albendazole $10 \mathrm{mg} / \mathrm{kg}$ one week before procedure and patients continue this treatment 3 months after.

In traditional PAIR one scolicidal agent is used after puncture and aspiration using an $18 \mathrm{G}$ needle. In our study we injected and aspirated subsequently two scolicidal agents: Hypertonic saline serum 20\% first, then absolute alcohol $96 \%$. We waited $15 \mathrm{~min}$ in each injection. Saline serum is more effective scolicidal effect, and facilitates the separation of endocyst from pericyst. Absolute alcohol has a sclerosing effects on the wall of the cyst. We believe that double PAIR increase effectiveness and reduces risks of recurrence in long term.

An $18 \mathrm{G}$ needle is used to puncture the cyst in the standard PAIR treatment. The needle tip can be plugged during aspiration of the cyst contents or it can remove from the cyst. To minimize all these disadvantage in our technique; we used $7 \mathrm{~F}$ drainage catheters to puncture the large cysts (more than $5 \mathrm{~cm}$ ). The distal tip of the catheter was like a pig's tail when we placed it into the cysts; so, it was stable in cysts during the procedure and obstruction of the catheter was minimized because there were multiple holes in the tip of the catheter.

Technically, rate success with PAIR varies between $88 \%$ and $100 \%$.The recurrence rate is between $1.6 \%$ and $10.9 \%$, anaphylaxia $0.03 \%$,while the morbidity rate varies between $25.2 \%$ and $32 \%$, including cyst infection (22\%) and biliary fistulae $(8.9 \%)$. Mortality is so low that can be negligible [11$13]$. Our success rate reaches $100 \%$ with no morbidity.

Length of hospitalization depends on whether a drainage catheter is used or not, and then the length of the stay may varies from 2.1 to 8 days, according to documented sources [11-13]. We opted, in our report, for short hospitalisation between $12 \mathrm{~h}$ and $24 \mathrm{~h}$, because all our cases were non-complicated.

\section{CONCLUSION}

Double PAIR is a minimally invasive procedure for management of active hydatid cyst in 
liver type I, II and III of Gharbi's classification. It's a safety, cost-effectiveness and more accurate procedure than simple PAIR. The association with oral albendazole increase efficacy and reduce recurrence risk, reduce length of hospitalization. We observed a high success rate with lower complication and we advise this technique for hydatid cyst Gharbi I, II and III.

\section{REFERENCES}

1. Acunas B, Rozanes I, Celik L. Purely cystic hydatid disease of the liver: treatment with percutaneous aspiration and injection of hypertonic saline. Radiology. 1992;182:541-3.

2. Akhan O, Ozmen MN, Dincer A, Sayek I, Gocmen A. Liver hydatid disease: long-term results of percutaneous treatment. Radiology. 1996;198:259-64.

3. WHO Informal Working Group on Echinococcosis. Guidelines for treatment of cystic and alveolar echinococcosis in humans. Bull World Health Organ. 1996; 74:231-242.

4. Filice C, Pirola F. A new therapeutic approach for hydatid liver cysts: aspiration and alcohol injection under sonographic guidance. Gastroenterology. 1990;98: 1366-8.

5. Gargouri M, Amor NB, Chehida FB, Hammou A, Gharbi HA, Cheikh MB, Kchouk H, Ayachi K, Golvan JY. Percutaneous treatment of hydatid cysts (Echinococcus granulosus). Cardiovascular and interventional radiology. 1990 May;13(3):169-73.

6. Gharbi HA, Hassine W, Brauner WB. Ultrasound examination of hydatid liver. Radiology. 1981;139:459- 63 .
7. Khuroo MS, Zargar SA, Mahajan R. Echinococcusgranulosus cysts in the liver: management with percutaneous drainage. Radiology. 1991;180:141-5.

8. Etlik O, Arslan H, Bay A, Sakarya ME, Harman M, Temizoz O, Kayan M, Bakan V, Unal O. Abdominal hydatid disease: long- term results of percutaneous treatment. Acta Radiologica. 2004 Jul 1;45(4):383-9.

9. Akhan O, Yildiz AE, Akinci D, Yildiz BD, Ciftci $T$. Is the adjuvant albendazole treatment really needed with PAIR in the management of liver hydatid cysts? A prospective, randomized trial with short-term follow-up results. Cardiovasc Intervent Radiol. 2014;37(6):1568-74.

10. Sayek I, Cakmakc1 M. Prophylactic effect of albendazole in experimental abdominal hydatidosis. Hepatogastroenterology 1992;39:424-6.

11. Smego RJ, Bhatti S, Khaliq AA, Beg MA. Percutaneous aspirationinjection-reaspiration drainage plus albendazole or mebendazole for hepatic cystic echinococcosis: a meta-analysis. Clin Infect Dis. 2003;37(8):1073-83.

12. Golemanov B, Grigorov N, Mitova R, Genov J, Vuchev D, Tamarozzi F, Brunetti E. Efficacy and safety of PAIR for cystic echinococcosis: experience on a large series of patients from Bulgaria. The American journal of tropical medicine and hygiene. 2011 Jan 5;84(1):48-51.

13. Yasawy MI, Mohammed AE, Bassam S, Karawi MA, Shariq S. Percutaneous aspiration and drainage with adjuvant medical therapy for treatment of hepatic hydatid cysts. World J Gastroenterol. 2011;17(5):646-50. 